\title{
Differential Effects of Fornix and Caudate Nucleus Lesions on Two Radial Maze Tasks: Evidence for Multiple Memory Systems
}

\author{
Mark G. Packard, Richard Hirsh, and Norman M. White \\ Departments of Psychology and Psychiatry, McGill University, Montreal, Quebec H3A 1B1, Canada
}

The present experiments were designed to examine the hypothesis that the mammalian brain contains anatomically distinct memory systems. Rats with bilateral lesions of caudate nucleus or fimbria-fornix and a control group were tested postoperatively on 1 of 2 versions of the radial maze task. In a standard win-shift version, each of the 8 arms of the maze was baited once, and the number of errors (revisits) in the first 8 choices of each trial was recorded. Fimbriafornix rats were impaired in choice accuracy, while caudate animals were unimpaired relative to controls. Different groups of rats with similar lesions were tested on a newly developed win-stay version of the radial maze, in which the location of 4 randomly selected baited arms was signaled by a light at the entrance to each arm, and which required rats to revisit arms in which reinforcement had been previously acquired within a trial. Rats with fimbria-fornix lesions were superior to controls in choice accuracy on the win-stay radial maze task, while caudate animals were impaired relative to controls. The results demonstrate a double dissociation of the mnemonic functions of the hippocampus and caudate nucleus. Some implications of the presence of 2 memory systems in the mammalian brain are discussed.

Hippocampal damage in rats causes deficits in the acquisition of various learning tasks (Hirsh, 1974; O'Keefe and Nadel, 1978; Olton, 1978), while sparing the acquisition of others (Silveria and Kimble, 1968; Harley, 1972; Samuels, 1972; Eichenbaum et al., 1986). The types of tasks in which learning is spared following hippocampal damage typically involve the consistent performance of a single response in the presence of a specific sensory cue. The literature includes numerous examples of learning tasks that fit this description and are spared by hippocampal lesions. One such task is simultaneous brightness discrimination (Silveria and Kimble, 1968; Harley, 1972; Samuels, 1972). In this task, animals must consistently perform the same approach response to the positive discriminative cue. Another example is successive cue go/no go olfactory discrimination (Eichenbaum et al., 1988). In this task, animals are successively presented with individual odor stimuli paired with reinforcement (i.e., go trials) or nonreinforcement (i.e., no go trials). A

\footnotetext{
Received Oct. 19, 1987; revised Aug. 23, 1988; accepted Sept. 19, 1988

This research was supported by grants from the Natural Sciences and Engineering Council of Canada to R. H., and from Fonds FCAR, Province of Quebec, to N.M.W.

Correspondence should be addressed to Mark G. Packard, Department of Psychology, McGill University, 1205 Dr. Penfield Ave., Montreal, Quebec H3A 1B1, Canada.

Copyright (c) 1989 Society for Neuroscience $0270-6474 / 89 / 051465-08 \$ 02.00 / 0$
}

response tendency to approach the positive odor stimulus is gradually acquired.

In contrast, tasks in which hippocampal animals are impaired do not involve the consistent performance of a single response to a specific discriminative cue. An example of such a learning task is the standard radial maze paradigm (Olton and Samuelson, 1976). In this task, animals obtain food pellets from multiple spatial locations, and accurate performance requires both memory for those maze arms previously visited and a tendency not to revisit arms within a trial.

Despite the ability of hippocampal animals to learn various tasks, there are few studies directly examining the neural basis of this spared learning. One candidate structure that may selectively serve this function is the caudate nucleus. The involvement of the caudate nucleus in memory is clearly indicated by studies showing that posttraining intracaudate electrical stimulation disrupts memory in a time-dependent manner (Wyers et al., 1968; Peeke and Herz, 1971; Wyers and Deadwyler, 1971). Furthermore, lesions of the caudate nucleus impair the acquisition of several operant tasks, the learning of which is typically unaffected by hippocampal lesions (see O'Keefe and Nadel, 1978). These include various avoidance tasks (Neill and Crossman, 1971; Mitcham and Thomas, 1972; Allen and Davison, 1973; Kirby and Polgar, 1974; Winocur, 1974; Prado-Acala et al., 1975), brightness discrimination (Schwartzbaum and Donovick, 1968), cued Morris water maze performance (Whishaw and Kolb, 1984; Whishaw et al., 1985), and visual/olfactory conditioned emotional responses (Viaud and White, 1989).

One problem in identifying brain structures that may selectively mediate different types of learning is the difficulty inherent in comparing results across different behavioral tasks. To overcome this problem in the present study we used 2 radial maze learning tasks. One was the standard "win-shift" paradigm (Olton and Samuelson, 1976), a task in which no single stimulus is consistently paired with the correct response. Acquisition of this task is reliably impaired following hippocampal system damage (see Olton, 1978).

In addition, we developed a new "win-stay" (Olton and Schlosberg, 1978), task for the radial maze in which a single stimulus was consistently paired with the correct response. The acquisition of this task involved learning an approach response to a specific sensory cue (light). Furthermore, animals were required to repeat choices to each lit maze arm within a trial. We hypothesized that the acquisition of this task would be impaired by lesions of the caudate nucleus.

In summary, by comparing behavior in the win-shift and winstay radial maze tasks, we sought to establish a double dissociation of hippocampal and caudate function in 2 learning tasks 
with the same motivational and motoric characteristics but in which the mnemonic requirements may differ.

\section{Materials and Methods}

Subjects. The subjects were 52 male I ong-Fvans rats $(275-325 \mathrm{gm})$ individually housed in a temperature-controlled $12 \mathrm{hr}$ light/dark environment and provided with access to water.

Apparatus. The apparatus was an elevated $(60 \mathrm{~cm}), 8$-arm radial maze made of wood and painted flat gray. The center platform was $40 \mathrm{~cm}$ in diameter, and each arm measured $60 \times 9 \mathrm{~cm}$. Food cups were drilled in to the floor at the end of each arm. Small, $6 \mathrm{~W}$ light bulbs were altached to a $3 \times 9 \mathrm{~cm}$ wood strip above the entrance to each of the 8 arms. The lights faced away from the center platform, and were controlled by a manual switchbox. A system of overhead tubes ran from the experimenter's location to the food cup at the end of each arm, allowing for rapid, unobtrusive rebaiting. The maze was surrounded on all 4 sides by dark blue curtains. It was centrally located so that opposing arms were equidistant from the curtains. A slanted overhead mirror was used to observe the animals from outside the curtains. Dim illumination was provided by overhead lights.

Surgery and histology. Prior to surgery, animals were each handled for $5 \mathrm{~min}$ a day during the first $10 \mathrm{~d}$ after their arrival in the laboratory.

Each rat was anesthetized with $60 \mathrm{mg} / \mathrm{kg}$ sodium pentobarbitol and placed in a stereotaxic instrument adjusted with skull flat. As several studies have revealed functional heterogeneity of the caudate nucleus in learning and memory (Winocur, 1974; Kirby and Polgar, 1974; Viaud and White, 1989), large bilateral lesions were made at both anterior and posterior caudate sites in order to produce maximum damage. Stereotaxic coordinates for anterior caudate lesions were $\mathrm{AP}=+1.5 \mathrm{~mm}$ from bregma, $\mathrm{ML}=+2.8 \mathrm{~mm}, \mathrm{DV}=-6.2 \mathrm{~mm}$ from skull surface. For posterior caudate lesions, coordinates were $\mathrm{AP}=+0.2 \mathrm{~mm}$ from breg$\mathrm{ma}, \mathrm{ML}=+4.3 \mathrm{~mm}, \mathrm{DV}=-6.7 \mathrm{~mm}$ from skull surface. At the anterior caudate site, $5 \mathrm{~mA}$ of direct current was passed for $20 \mathrm{sec}$ through an electrode insulated except for $0.8 \mathrm{~mm}$ at the tip. At the posterior site, $4 \mathrm{~mA}$ was passed for $15 \mathrm{sec}$. After making lesions on one side, a 2 week recovery period was allowed before making the lesions on the other. All rats were fed a supplement of rat chow mash daily during recovery periods. Behavioral testing was begun 2 weeks after the second lesion was made. None of the caudate animals were aphagic or adipsic at the time of testing.

For fimbria-fornix animals, stereotaxic coordinates were $\mathrm{AP}=-1.3$ $\mathrm{mm}$ from bregma, $\mathrm{ML}=1.2 \mathrm{~mm}$, and $0.5 \mathrm{~mm}, \mathrm{DV}=-4.6 \mathrm{~mm}$, and $-4.2 \mathrm{~mm}$ from skull surface. Four lesions were made across the width of the fimbria-fornix by passing $3 \mathrm{~mA}$ of current for $5 \mathrm{sec}$ at each site through an electrode insulated except for $0.8 \mathrm{~mm}$ at the tip. Behavioral testing was begun following a 2 week postoperative recovery period.

Following testing, all animals with lesions were deeply anesthetized with a 1 cc injection of $30 \%$ chloral hydrate solution and perfused with physiological saline followed by $10 \%$ formol-saline. The brains were removed and fixed in 10\% formol-saline prior to being sectioned. Brains were sectioned at $20 \mu \mathrm{m}$, and every fifth section was mounted and stained using the Kleuver-Berea method.

In order to ensure similarity of the lesions in the animals tested in both tasks, a set of criteria for histological examination prior to including animals in the final data analyses was used. Inclusion in caudate groups required extensive damage at both the anterior and posterior lesion sites, with no damage to septal nuclei medial to the caudate. Damage to the dorsal lateral septal nucleus eliminated one animal from the winshift task for the caudate group. Inclusion in fornix groups required damage to fimbria-fornix posterior to the septum and anterior to the dorsal hippocampus. Direct damage to the dorsal hippocampus eliminated one animal from the win-shift task, while damage to the septum eliminated one animal from the win-stay task group.

The histological material from the animals included in the analyses of the behavioral data is illustrated in Figure 1, with the extent of maximal and minimal damage in each lesion group and task shown. In caudate animals, the antcrior lesions produccd dorsal-medial caudate damage ranging from AP 1.7 to $0.2 \mathrm{~mm}$ from bregma. Posterior lesions produced damage to ventral-lateral caudate, ranging from AP 0.1 to $-1.3 \mathrm{~mm}$ from bregma. In animals in both experiments, bilateral caudate damage was extensive, with the average lesion destroying approximately $80 \%$ of the head of the caudate. Damage to surrounding structures varied slightly across behavioral groups, with occasional incomplete damage to internal capsule, claustrum, and globus pallidus. Extracau-

\section{WIN - STAY TASK \\ WIN - SHIFT TASK \\ Caudate \\ Lesions}
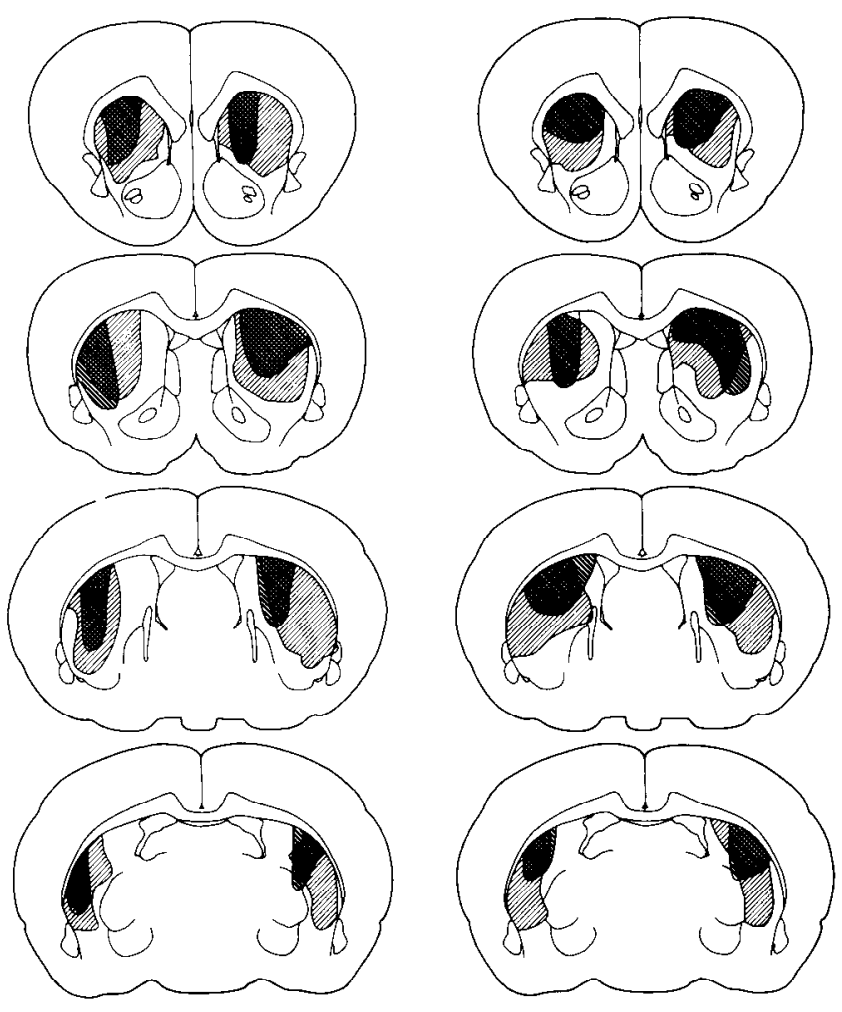

FORNIX

LESIONS
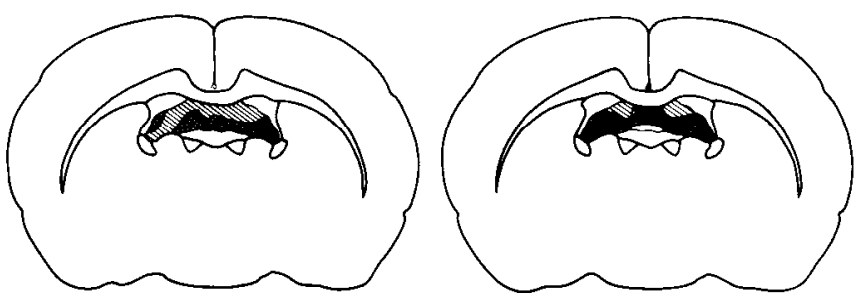

Figure 1. Coronal sections showing the minimum (dark hatching), and maximum (dark + light hatching) extent of caudate lesions and fimbriafornix lesions in both tasks. For caudate lesions, slices are shown at 1.0 $\mathrm{mm}$ intervals ranging from $+1.7 \mathrm{~mm}$ to $-1.3 \mathrm{~mm}$ from bregma; fimbria-fornix lesions are shown at $-1.3 \mathrm{~mm}$ from bregma. (Atlas of Paxinos and Watson, 1982.)

date damage did not correlate with behavioral results. None of the caudate lesions damaged the anterior commissure or septal nuclei.

Fimbria-fornix lesions (Fig. 1), in both experiments ranged from AP -1.0 to $-1.4 \mathrm{~mm}$ from bregma. All animals suffered damage to the fimbria-fornix posterior to the septum and anterior to the dorsal hippocampus. The average fornix lesion destroyed approximately $85 \%$ of the fimbria-fornix complex at the section shown in Figure $2(-1.3 \mathrm{~mm}$ from bregma). In two animals that sustained partial lesions, there was some sparing of the medial fimbria-fornix complex.

\section{Experiment 1}

In this experiment, animals with caudate and fimbria-fornix lesions were tested on a radial maze task in which correct performance depended on win-stay behavior. A specific sensory cue (light) was consistently paired with the correct response, and animals were required to revisit maze arms within a trial. 


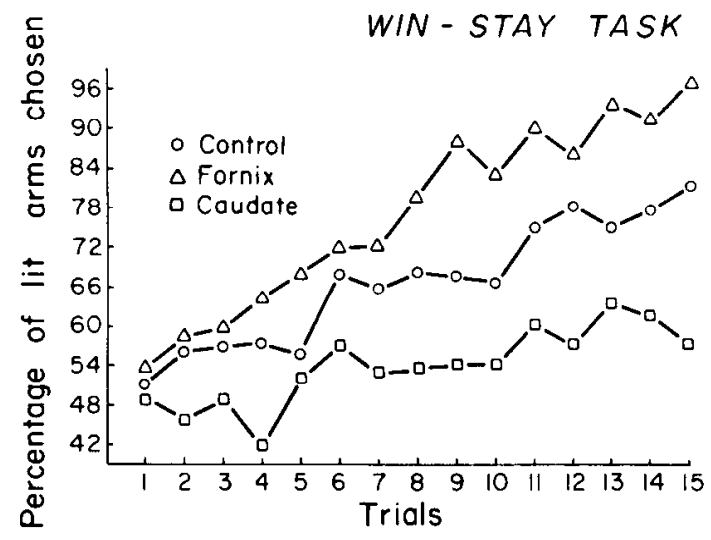

Figure 2. Percentage (lit arms/total arms $\times 100$ ) of correct arms chosen by each group during the 15 acquisition trials on the win-stay radial maze task.

\section{Methods}

Subjects. Animals were assigned to 1 of 3 groups: caudate $(n=9)$, fimbria-fornix $(n=7)$, and unoperated control $(n=9)$. The number of subjects in each group refers to animals that met the histological criteria.

Procedure. Prior to testing, all animals were reduced to $85 \%$ of their ad lib feeding weights. For preliminary training, each rat was individually placed on the center platform of the maze for $5 \mathrm{~min}$ on 2 consecutive days. No food was present in the maze during this habituation period. On day 3 , food trials began. On each food trial, 4 randomly selected arms were lit and baited. After a rat had visited one of the lit arms, it was rebaited. After a second visit to the same arm, its light was turned off and no further food was placed in that arm. Thus, animals were required to visit each of the 4 lit arms twice, earning 8 pellets within a trial. Animals were removed from the maze after 8 pellets had been earned or $10 \mathrm{~min}$ had elapsed. Records were kept of the arms entered and the order of entry. Visits to unlit arms were scored as errors. Food-rewarded trials were run once a day for $15 \mathrm{~d}$. On each day 4 randomly selected arms were lit and baited.

Probe trials, which were designed to provide a more sensitive measure of win-stay behavior, were run on days 16-19. During these trials, rats were placed on the maze with only a single arm lit and baited. Following a visit to that arm, its light was turned off and another arm was immediately lit and baited. This procedure continued until $8 \mathrm{lit} /$ baited arms had been selected. The 8 arms baited during these trials were randomly selected prior to each trial, and thus some arms were lit more than once within a probe trial. Visits to unlit arms were scored as errors.

\section{Results of experiment 1}

Learning curves for the acquisition of win-stay behavior in the radial maze are presented in Figure 2. Animals in all groups earned 8 pellets within the $10 \mathrm{~min}$ allotted, and so the data are presented as the pereentage of lit arms chosen per trial. As shown in Figure 2, the performance of caudate animals was impaired relative to controls. In contrast, the performance of fimbriafornix rats was superior to that of controls. A 2-way 1-repeated measure ANOVA was computed on the data shown in Figure 2. The analysis revealed a significant main effect of lesion $[F(2,20)$ $=18.54, p<0.01]$. In addition, a significant main effect of trial revealed that all groups improved over trials $[F(14,280)=13.91$, $p<0.01]$. Tukey's post hoc tests revealed that caudate animals were impaired relative to controls in choice accuracy $(Q=3.42$, $p<0.05$ ). Similar tests revealed that fimbria-fornix rats were superior to both control $(Q=3.39, p<0.05)$ and caudate $(Q$ $=4.11, p<0.05$ ) animals in choice accuracy.

Results from the probe trials are illustrated in Figure 3. Animals selecting arms at random would display a choice accuracy of $12.5 \%$, since only 1 of $8 \mathrm{arms}$ was lit/baited at a time. As

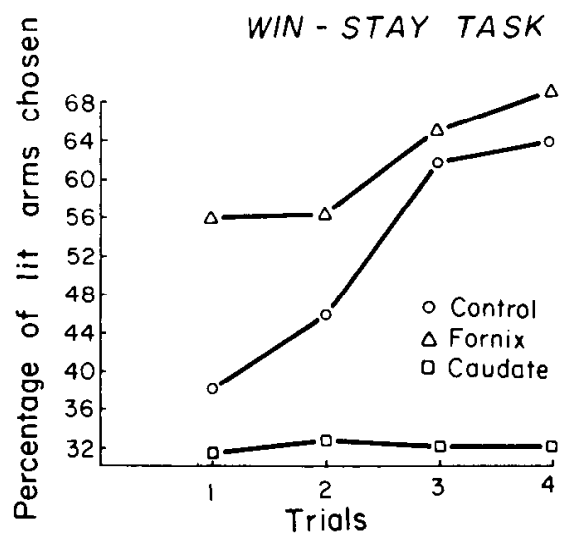

Figure 3. Percentage (lit arms/total arms $\times 100$ ) of correct arms chosen by each group during each of 4 probe trials run on days $16-19$, following completion of the 15 acquisition trials of win-stay radial maze behavior.

seen in Figure 3, animals in all groups selected lit arms at a level greater than chance, however, the differences in choice accuracy observed during the 15 acquisition trials were maintained during the probe trials. Thus, caudate animals were impaired relative to controls, whilc fornix animals were superior in choice accuracy. A 2-way 1-repeated measures ANOVA was computed on the data shown in Figure 3. The analysis revealed a significant interaction $[F(6,66)=4.06, p<0.01]$. In addition, there was a highly significant main effect of lesion $[F(92,66)=111.2, p<$ $0.01]$. Tukey's post hoc tests revealed that caudate animals were significantly impaired relative to both control $(Q=7.34, p<$ $0.05)$ and formix $(Q=10.78, p<0.05)$ animals. As observed in acquisition trials, fornix rats were also superior to control rats in choice accuracy during probe trials $(Q=3.43, p<0.05)$.

Response distribution analyses were computed on the final (15th) trial of acquisition to provide a measure of the degree of win-stay and win-shift behavior exhibited by animals in all groups. For each animal, the percentage of visits to each arm was computed and then rank-ordered according to frequency (1-8) of visits. The group mean percentage of visits to each arm was then computed. Animals performing at 100\% accuracy in the win-stay task would visit each of the 4 lit arms twice within a trial ( $25 \%$ of total visits), while ignoring the 4 unlit arms. As shown in Figure 4, both control and fimbria-fornix rats displayed a high degree of win-stay behavior. In contrast, caudate rats distributed their responses more evenly over the 8 choices, displaying a high degree of win-shift behavior. A 1-way ANOVA computed on the slopes of the frequency distributions for each group revealed that the slope for the fimbria-fornix group was significantly different from that for the caudate and control animals $[F(2,24)=41.35, p<0.01]$.

\section{Discussion of experiment 1}

The results of experiment 1 demonstrate differential effects of caudate nucleus and fimbria-fornix lesions on win-stay radial maze performance. Caudate nucleus lesions impaired acquisition, while fimbria-fornix lesions facilitated acquisition. The impairment observed in the caudate animals suggests that the caudate nucleus is involved in the acquisition of tasks that may be acquired through the formation of an association between a specific sensory cue and a single response tendency.

This general interpretation may also account for caudateinduced deficits that have been observed in several other tasks, 
including various avoidance paradigms (Neill and Crossman, 1971; Allen et al., 1972; Mitcham and Thomas, 1972; Allen and Davison, 1973; Winocur, 1974; Prado-Acala et al., 1975), cued Morris water maze performances (Whishaw and Kolb, 1984; Whishaw et al., 1987), brightness discrimination (Schwartzbaum and Donovick, 1968), right-left maze discrimination (Cook and Kesner, 1984), and visual/olfactory conditioned emotional responding (Viaud and White, 1989).

In contrast to the impairment observed following lesions of the caudate nucleus, animals with fimbria-fornix lesions acquired the win-stay task more quickly than normal animals. Other learning paradigms in which hippocampal/fornix lesions have similar effects include various avoidance tasks (see O'Keefe and Nadel, 1978), cue-guided lever press alternation (Stevens and Cowey, 1972), 2-bar alternation (Jackson and Strong, 1969), single-bar go/no go alternation (Means et al., 1970; Walker et al., 1970), olfactory discrimination reversal (Eichenbaum et al., 1986; Fagan and Olton, 1986), and successive cue go/no go olfactory discrimination (Eichenbaum et al., 1988).

An important factor in understanding the apparent facilitation of win-stay behavior in the radial maze following fimbria-fornix lesions is the predisposition of normal rats to win-shift in this apparatus. When normal rats were given the opportunity to use either win-shift or win-stay tendencies in the radial maze (i.e., both tendencies were equally rewarded), they tended to winshift (Olton and Schlosberg, 1978; see also Gaffan and Davies, 1981; Evenden and Robbins, 1986; Gaffan and Eacott, 1986). In the present experiment the acquisition of the win-stay task by normal rats involved a phase in which predominant winshift tendencies were present and overcome. Olton (1978) has suggested that win-shift tendencies are mediated by the hippocampus. In the absence of a functional hippocampal system, the fimbria-fornix rats in the present experiment may have been less likely to exhibit win-shift tendencies and so performed better than normal rats on the win-stay task.

Results from the response distribution analyses also support this interpretation. Lesions of the caudate nucleus led to persistent win-shift bchavior, although the reinforcement contingencies required animals to repeat previous choices. In contrast, as shown by the response distribution, fornix lesions facilitated approach responses to the lit arms; that is, the influence on behavior by stimulus-response tendencies was facilitated.

This finding is consistent with results from several behavioral paradigms in which hippocampal deficits have been attenuated by the introduction of a specific sensory cue, including complex T-maze behavior (Winocur and Breckenridge, 1973), DRL operant responding (Pelligrino and Clapp, 1971; Rickert et al., 1973; Gray and Sainsbury, 1979), Morris water maze performance (Sutherland et al., 1982), and food/water finding tasks (O'Keefe et al., 1975; O'Keefe and Dunway, 1980).

In summary, the results of experiment 1 suggest that the caudate nucleus mediated learning in which a specific sensory cue was consistently paired with the correct response. Lesions of the fimbria-fornix faciliated learning this behavior. The fact that damage to the caudate and hippocampus had opposite effects on the acquisition of this task suggests that these two structures may be involved in memory systems that are specialized for learning different types of behaviors.

\section{Experiment 2}

To complete the double dissociation of hippocampal and caudate memory function, this experiment examined the effect of

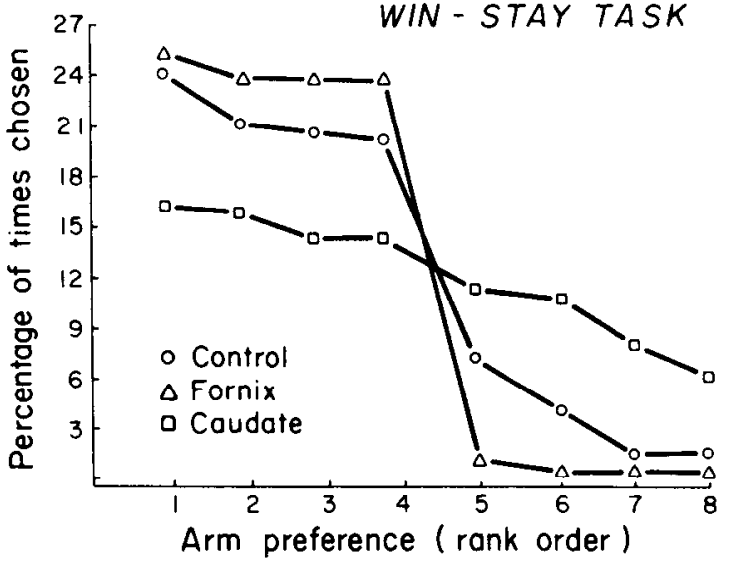

Figure 4. Response distribution analyses for the win-stay radial maze task. The analyses provide a measure of the degree of shift and stay behavior displayed by each group.

fimbria-fornix and caudate nucleus lesions on win-shift behavior in the standard radial maze task (Olton and Samuelson, 1976). Several previous studies have demonstrated that fornix/ hippocampal lesions impair this type of radial maze behavior (Olton, 1978; Becker et al., 1980). However, the effect of caudate damage on the acquisition of this task is controversial. Studies have reported either no effect (Becker et al., 1980; Cook and Kesner, 1984; Volpe et al., 1986) or a slight disruption in performance (Winocur, 1980; Masuda and Iwasaki, 1984). It is well established that normal animals select correct arms in the radial maze on the basis of their spatial location relative to discriminative extra-maze stimuli (Olton, 1978; Suzuki et al., 1980). It has been suggested that disruption of win-shift radial maze behavior following caudate lesions may occur when the extramaze environment is relatively devoid of cues (Masuda and Iwasaki, 1984). The present study, which used the same impoverished extramaze environment as in experiment 1 , also represents a test of this hypothesis.

\section{Methods}

Subjects. Animals were assigned to 1 of 3 groups: caudate $(n=8)$, fimbria-fornix $(n=8)$, and unoperated control $(n=8)$. The numbers of subjects in each group refers to animals that met the histological criteria described.

Procedure. Preliminary training without food was identical to that described in experiment 1 . On day 3 , each rat was individually placed on the maze. A single $45 \mathrm{mg}$ food pellet was available in the food cup of each of the 8 arms. On the initial food trial only, 4 pellets were also placed on the center platform. Animals were removed from the maze after 8 pellets had been retrieved from the food cups or $10 \mathrm{~min}$ had elapsed. Records were kept of the arms entercd and the order of entry. Food-rewarded trials were run once a day until all control animals had reached a criterion of selecting 7 different arms in the first 8 choices on 2 consecutive days.

\section{Results of experiment 2}

As illustrated in Figure 5, caudate animals showed no deficit in the acquisition of win-shift radial maze behavior relative to controls. All animals in both the caudate and control groups reached the criterion of selecting 7 different arms in the first 8 choices on 2 consecutive days within 4 testing days. In contrast, fimbria-fornix animals were impaired relative to controls. A 2-way, 1-repeated measure ANOVA was computed on the data in Figure 5. The analysis revealed no significant interaction 
WIN-SHIFT TASK

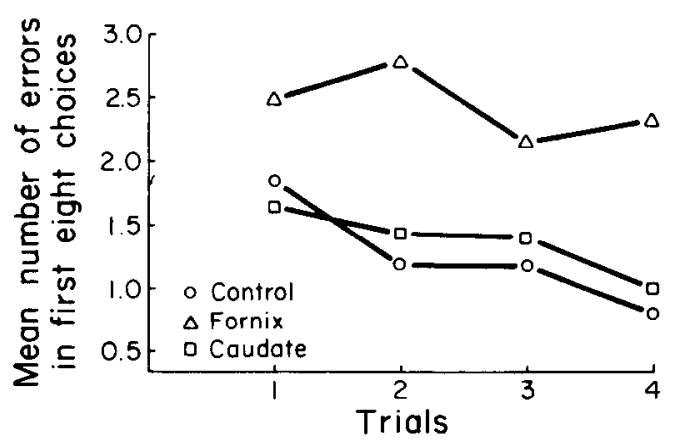

Figure 5. Mean number of errors in the first 8 choices during acquisition of win-shift radial maze behavior.

effect. There was a significant overall main effect of lesion $[F(2,63)$ $=93.02, p<0.01]$. In addition, a significant main effect of trial $[F(3,63)=5.2, p<0.01]$ revealed that all groups improved over trials. Tukey's post hoc tests revealed that fimbria-fornix lesions produced a statistically reliable deficit in choice accuracy (as measured by the number of errors in the first 8 choices of each trial) compared with control rats $(Q=6.38, p<0.05)$. Caudate animals were not significantly different from controls in choice accuracy $(Q=1.09$, n.s. $)$.

Response distribution analyses similar to those computed in experiment 1 were also computed on all 4 acquisition trials in the present experiment. Animals producing $100 \%$ win-shift behavior would visit each of the 8 arms once (12.5\% of total visits), within a trial. As shown in Figure 6, control and caudate rats distributed their choices equally among the 8 arms, indicating a high degree of win-shift behavior. In contrast, fimbria-fornix rats made several visits to arms in which reinforcement had previously occurred, suggesting a tendency to win-stay. A 1-way ANOVA computed on the slopes of the frequency distribution for each group revealed that the slope of the fimbria-fornix animals was significantly greater than that of caudate and control rats $[F(2,23)=7.02, p<0.01]$.

\section{Discussion of experiment 2}

These results are in agreement with previous studies that have demonstrated deficits in win-shift radial maze performance following fornix/hippocampal lesions (Olton, 1978; Becker et al., 1980). In contrast, lesions of the caudate nucleus had no effect on win-shift radial maze performance. This finding is also in agreement with some previous reports (Becker et al., 1980; Cook and Kesner, 1984; Volpe et al., 1986).

The results of the response distribution analysis raise an interesting point concerning the nature of the mnemonic deficit underlying the poor win-shift performance of the rats with fornix lesions. It has been suggested that these animals are deficient because their "working memory" (Olton and Papas, 1979)their capacity to remember which arms of the maze they have already visited within a trial-is impaired. In the absence of working memory, fornix animals should choose randomly among the 8 arms on every choice of a given trial. Therefore, errors (revisits) should be distributed randomly, and the frequency distribution curve should be flat. Our results (Fig. 6), although computed on a relatively small number of trials, suggest that the errors made by fornix animals may not be randomly distributed. Instead, individual fornix rats displayed preferences

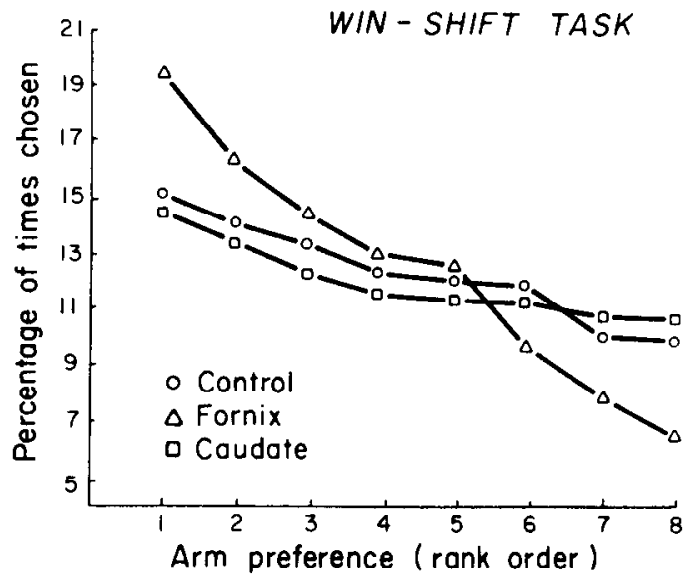

Figure 6. Response distribution analyses for the win-shift radial maze task. The analyses provide a measure of the degree of shift and stay behavior displayed by each group during acquisition.

for particular maze arms. Although a loss of working memory may explain the inability of fornix rats to win-shift accurately, the development of such preferences suggests that in the absence of a functional hippocampal/fornix system, behavior is increasingly controlled by a learning system that favors win-stay behavior. This is the prediction that would be made on the assumption that the behavior of these animals is controlled in part by the caudate nucleus, which mediates win-stay radial maze performance (experiment 1).

Two studies have reported a slight disruptive effect of caudate lesions on radial maze choice accuracy (Winocur, 1980; Masuda and Iwasaki, 1984), although the magnitude of the deficit was not as severe as that seen following hippocampal damage in the same studies. It has been suggested that an impairment in winshift radial maze performance following caudate damage may appear in environments that are relatively devoid of extramaze cues (Masuda and Iwasaki, 1984). However, in the present study, an impoverished extramaze environment did not impair the choice accuracy of caudate animals relative to controls. Furthermore, given the large lesions made in the present study, the inability of caudate damage to impair win-shift radial maze behavior appears to be a reliable effect.

In summary, the results of experiment 2 confirm that an intact hippocampal system is necessary for accurate win-shift behavior in the radial maze, a lask that requires memory for the presence or absence of food in multiple spatial locations. In contrast, lesions of the caudate nucleus, which impaired the acquisition of win-stay radial maze behavior (experiment 1 ), had no effect on win-shift performance.

\section{General Discussion}

Taken together, the results of experiments 1 and 2 demonstrate a double dissociation of learning and memory systems involving the hippocampus and caudate nucleus. These findings suggest that there may be at least 2 such systems operating in the normal brain.

An anatomical dissociation of mammalian learning systems similar to the one suggested by the present data has been revealed in studies using nonhuman primates and human amnesics. In monkeys, the acquisition of a long-term visual discrimination task (in which reinforcement is paired with the same stimuli across all trials) is impaired by lesions of the caudate 
nucleus (Buerger et al., 1974). Lesions of the temporal stem, which connects the inferior temporal cortex with structures including the caudate nucleus, also disrupts the acquisition of visual discrimination learning in monkeys (Zola-Morgan et al., 1982). In contrast, the acquisition of a delayed non-match-tosample task (DNMS), employing trial unique stimuli is unaffected by lesions of the temporal stem (Zola-Morgan et al., 1982). DNMS acquisition is disrupted by lesions of the hippocampus/amygdala, while long-term visual discrimination is unaffected (Gaffan, 1974; Zola-Morgan et al., 1982; Malamut et al., 1984; see also Gaffan et al., 1984a-c).

In humans, recent studies characterizing the amnesic syndrome following presumed damage to the caudate nucleus in cases of Huntington's disease versus presumed damage to cortical/limbic structures including the hippocampus in cases of Alzheimer's disease also suggest that these structures mediate different types of memory processes. For example, independent of their level of motor dysfunction, Huntington's patients were impaired in the acquisition of a pursuit rotor task that involved learning a motor response to a rotating stimulus. Patients with Alzheimer's disease acquired this task normally (Heindel et al., 1988). In contrast. Alzheimer's patients were impaired relative to Huntington's patients in immediate and delayed recall on a verbal recognition span memory test (Heindel et al., 1988). A similar distinction has been made by Martone et al. (1984). Therefore, a dissociation between the mnemonic functions of the hippocampal and caudate systems may generalize across mammalian species.

The hypothesis that the hippocampal and caudate systems mediate different types of learning raises 2 important questions. The first involves the nature of the interaction between the 2 systems in animals with intact brains. The second concerns identification of the critical operating principle(s) that distinguish between the 2 systems.

The data of the present study and those reviewed imply that more than one learning system may function simultaneously in the normal brain. The suggestion of simultaneous involvement of multiple memory systems is supported by electrophysiological studies demonstrating behavioral correlates of hippocampal unit activity during the acquisition of tasks that are unaffected by hippocampal lesions (Solomon and Moore, 1975; Eichenbaum et al., 1987; see also Berger and Orr, 1982; Ross et al., 1984; Port et al., 1987).

The ultimate expression of multiple memory systems in behavior may depend on the nature of the task. For example, the facilitative effects of fornix lesions on win-stay behavior in the present study suggests that for some tasks the 2 systems may be in competition. The facilitation of successive cue go/no go olfactory discrimination following fornix lesions has similarly been interpreted as resulting from the "unnecessary application" of the hippocampal system by normal animals (Eichenbaum et al., 1988).

There are other tasks on which hippocampal and caudate lesions produce similar acquisition deficits. For example, hippocampal (see O'Keefe and Nadel, 1978) and caudate lesions impair the acquisition of various alternation tasks, including spontaneous (Cross et al., 1965; Kirkby, 1969a; Divac et al., 1975), spatial (Chorover and Gross, 1963; Mikulas, 1966; Butters and Rosvold, 1968; Divac, 1971; but see also Schwartzbaum and Donovick, 1968), and delayed alternation (Mikulas and Isaacson, 1965; Divac and Oberg, 1975). In addition, both hippocampal (see O'Keefe and Nadel, 1978), and caudate le- sions impair brightness discrimination reversal (Kirkby, 1969b) and spatial reversals (Divac, 1971; Hannon and Bader, 1974; Kolb, 1977; Mitchell et al., 1985). These findings suggest that in some situations the 2 systems may act synergistically to produce learned behavior.

The sccond question concerns the nature of the opcrating principle(s) that distinguish the 2 systems. Several sets of such principles have been proposed by investigators who have compared the tasks on which learning is debilitated with those on which learning is spared by lesions of the hippocampal system in animals and humans (Milner, 1962; Corkin. 1968: Hirsh. 1974; O'Keefe and Nadel, 1978: Olton and Papas, 1979: Cohen and Squire, 1980). In animals, these principles have been termed contextual retrieval versus habit (Hirsh, 1974), cognitive mapping versus taxon learning (O'Keefe and Nadel, 1978), memory versus habit (Mishkin and Petri. 1984), and working versus reference memory (Olton and Papas, 1979; see also Kesner and Dimattia, 1987; Thomas and Gash, 1985). In each case, the former of the 2 principles refers to the type of memory mediated by the hippocampus, and the latter refers to some unknown substrate. The data of the present study suggest that this substrate may involve the caudate nucleus.

The present study was not specifically designed to differentiate among these theories, and the results are, in fact, consistent with all of them. The standard win-shift radial maze task may involve spatial cognitive mapping (O'Keefe and Nadel, 1978), working memory (Olton and Papas, 1979), and/or contextual retrieval (Hirsh, 1974). Therefore, any of these descriptions may be applied to the hippocampal system when its destruction debilitates performance on this task. Similarly, the win-stay task used here may involve taxon learning (O'Keefe and Nadel. 1978), reference memory (Olton and Papas, 1979), and/or habit formation (Hirsh, 1974: Mishkin and Petri, 1984). Since caudate lesions impaired acquisition of this task, any of these descriptions can be applied to the function of the caudate system.

Until now, animal studies on the various dual-memory theories have been carried out solely on the basis of hippocampal function (O'Keefe and Nadel, 1978; Olton and Feustle. 1981; Walker and Olton, 1984). The identification of the caudate nucleus as a structure mediating at least some of the learning that is spared following hippocampal damage allows for experiments that can assess these theories by manipulating both substrates.

Although the present data do not necessitate a new dualmemory theory, they do provide an example of how the manipulation of 2 memory substrates can assist in revealing variables that may be important in understanding the difference between the 2 systems. One important variable on which the present radial maze tasks differ, and which as received little attention in previous studies, is the role that reinforcers play in learning. In the win-shift task, reinforcers do not act in the classical manner to increase the probability of making the most recently reinforced response (Thorndike, 1933; I full, 1943) since animals must learn to avoid previous reinforced choices within a trial. To solve this task, animals must learn about the stimulus attributes of the reinforcers in the situation, namely, the presence or absence of food at multiple spatial locations.

In contrast, the acquisition of the win-stay task can be understood in terms of the "classical" action of reinforcers: with each reinforced response the probability of approaching a lit alley was increased. Knowledge of the stimulus attributes of the reinforcer was not necessary for the acquisition of this task. Therefore, the present data suggest that one of the variables differ- 
entiating between the functions of the hippocampal and caudate memory systems may be the role played by reinforcers. This emphasis on the role of reinforcers in acquisition neither competes with nor replaces the existing dual-memory theories. Rather, consideration of the role of reinforcers provides an operationally defined experimental variable that may help to understand the operating principles of the 2 memory systems.

In summary, the present results provide empirical evidence for the existence of multiple memory systems in the mammalian brain. Future studies aimed at further delineating the critical operating principle(s) that distinguish these systems, and the extent and nature of the interaction between them, will advance our understanding of the neural basis of learning and memory.

\section{References}

Allen, J. D., and C. S. Davison (1973) Effects of caudate lesions on signaled and nonsignaled Sidman avoidance in the rat. Behav. Biol. 8: 239-250.

Allen, J. D., J. C. Mitcham, and J. I. Bryd (1972) Effects of caudate lesions on the acquisition and retention of Sidman avoidance in the rat. Psychonom. Sci. 27: 157-160.

Becker, J. T., J. A. Walker, and D. S. Olton (1980) Neuroanatomical basis of spatial memory. Brain Res. 200: 307-320.

Berger, T. W.. and W. B. Orr (1982) Role of the hippocampus in reversal learning of the rabbit nictitating membrane response. In Conditioning: Representation of Involved Neural Functions, C. D. Woody, ed., pp. 1-12, Plenum, New York.

Buerger, A. A., C. G. Gross, and C. E. Rocha-Miranda (1974) Effects of ventral putamen lesions on discrimination learning by monkeys. J. Comp. Physiol. Psychol. 86: 440-446.

Butters, N., and H. E. Rosvold (1968) Effect of caudate and septal lesions on resistance to extinction and delayed alternation. J. Comp. Physiol. Psychol. 65: 397-403.

Chorover, S. L., and C. G. Gross (1963) Caudate nucleus lesions: Behavioral effects in the rat. Science 141:826-827.

Cohen, N. J., and L. R. Squire (1980) Preserved learning and retention of pattern-analyzing skill in amnesia: Dissociation of knowing how and knowing that. Science 210: 207-209.

Cook, D. G., and R. P. Kesner (1984) Memory for egocentric spatial localization in an animal model of advanced Huntington's disease. Soc. Neurosci. Abstr. 10: 133.

Corkin, S. (1968) Acquisition of motor skill after bilateral medial temporal-lobe excision. Neuropsychologia 6: 255-265.

Divac, I. (1971) Frontal lobe system and spatial reversal in the rat. Neuropsychologia 9: 175-183.

Divac, I., and R. G. E. Oberg (1975) Dissociative effects of selective lesions in the caudate nucleus of cats and rats. Acta Neuro. Exp. 35: 647-659.

Divac, I., R. G. E. Wikmark, and A. Gade (1975) Spontaneous alternation in rats with lesions in the frontal lobes: An extension of the frontal lobe syndrome. Physiol. Psychol. 3: 39-42.

Eichcnbaum, H., A. Fagan, and N. J. Cohen (1986) Normal olfactory discrimination learning set and facilitation of reversal learning after medial-temporal damage in rats: Implications for an account of preserved learning in amnesia. J. Neurosci. 6: 1876-1884.

Eichenbaum, H., M. Kuperstein, A. Fagan, and J. Nagode (1987) Cuesampling and goal-approach correlates of hippocampal unit activity in rats performing an odor-discrimination task. J. Neurosci. 7: 716732.

Eichenbaum, H., A. Fagan, P. Matthews, and N. J. Cohen (1988) Hippocampal system dysfunction and odor discrimination learning in rats: Impairment or facilitation depending on representational demands. Behav. Ncurosci. 102: 331-339.

Evenden, J. L., and T. W. Robbins (1984) Win-stay behaviour in the rat. Q. J. Exp. Psychol. 36B: 1-26

ragan, A. M., and D. S. Olton (1986) Learning sets, discrimination reversal, and hippocampal function. Behav. Brain Res. 21: 13-20.

Gaffan, D. (1974) Recognition impaired and association intact in the memory of monkeys after transection of the fornix. J. Comp. Physiol. Psychol. 86: 1100-1109.
Gaffan, D., R. C. Saunders, E. A. Gaffan, S. Harrison, C. Sheilds, and M. J. Owen. (1984a) Effects of fornix transection upon associative memory in monkeys: Role of the hippocampus in learned action. Q. J. Exp. Psychol. 36B: 173-221.

Gaffan, D., E. A. Gaffan, and S. Harrison (1984b) Effects of fornix transection on spontaneous and trained non-matching by monkeys. Q. J. Exp. Psychol. 36B: 285-305.

Gaffan, D., C. Sheilds, and S. Harrison (1984c) Delayed matching by fornix-transected monkeys: The sample, the push and the bait. Q. J. Exp. Psychol. 36B: 305-317.

Gaffan, E. A., and J. Davies (1981) The role of exploration in winshift and win-stay performance on a radial maze. Learning Motivation 12: 282-299.

Gaffan, E. A., and M. Eacott (1986) Memory for feeding in rat's spatial and visual choice behaviour. Q. J. Exp. Psychol. 38B: 285-311.

Gray, D., S., and R. S. Sainsbury (1979) The effects of fimbria lesions on cue utilization in the rat. Physiol. Behav. 22: 325-329.

Gross, C. G., S. L. Chorover, and S. M. Cohen (1965) Caudate, cortical hippocampal, and dorsal thalamic lesions in rats: Alternation and Hebb-Williams maze performance. Neuropsychologia 3: 53-68.

Hannon, R., and A. Bader (1974) A comparison of frontal pole, anterior median and caudate nucleus lesions in the rat. Physiol. Behav. 13: 513-521.

Harley, C. W. (1972) impal lesions and two cue discrimination in the rat. Physiol.

Heindel, W., N. Butte J. Salmon (1988) Impaired learning of a motor skill in patients with Huntington's disease. Behav. Neurosci. 102: $141-150$.

Hirsh, R. (1974) The hippocampus and contextual retrieval of information from memory: A theory. Behav. Biol. 12: 421-444.

Hull, C. L. (1943) Principles of Behavior, Appleton-Century-Crofts, New York.

Jackson, W. J., and P. N. Strong (1969) Differential effects of hippocampal lesions upon sequential tasks and maze learning by the rat. J. Comp. Physiol. Psychol. 68: 442-450.

Kesner, R. P., and B. V. DiMattia (1987) Neurobiology of an attribute model of memory. Prog. Psychobiol. Physiolog. Psychol. 12: 207277.

Kirkby, R. J. (1969a) Caudate nucleus lesions impair spontaneous alternation. Percept. Motor Skills 29: 550.

Kirkby, R. J. (1969b) Caudate nucleus lesions and perserverative behavior. Physiol. Behav. 4: 451-454.

Kirkby, R. J., and S. Polgar (1974) Active avoidance in the laboratory rat following lesions of the dorsal or ventral caudate nucleus. Physiol. Psychol. 2: 301-306.

Kolb, B. (1977) Studies on the caudate-putamen and the dorsomedial thalamic nucleus of the rat: Implications for mammalian frontal-lobe functions. Physiol. Behav. 18: 237-244.

Malamut, B. L., R. C. Saunders, and M. Mishkin (1984) Monkeys with combined amygdalo-hippocampal lesions succeed in object discrimination learning despite 24 -hour intervals. Behav. Neurosci. 98 : 759-769.

Martone, M., N. Butters, M. Payne, J. Becker, and D. S. Sax (1984) Dissociations between skill learning and verbal recognition in amnesia and dementia. Arch. Neurology 41: 965-970.

Masuda, Y., and T. Iwasaki (1984) Effects of caudate lesions on radial naze behavior in rats. Jpn. Psychol. Res. 26: 42-49.

Means, L. W., D. W. Walker, and R. L. Isaacson (1970) Facilitated single-alternation go, no-go acquisition following hippocampectomy in the rat. J. Comp. Physiol. Psychol. 72: 278-285.

Mikulas, W. L. (1966) Effects of lights at the choice point on spatial alternation and position learning by normal rats and rats with bilateral lesions of the caudate nucleus. Psychonom. Sci. 5: 275-276.

Mikulas, W. L., and R. I. Isaacson (1965) Impairment and perseveration in delayed tasks due to bilateral lesions of the caudate nucleus. Psychonom. Sci. 3: 485-486.

Milner, B. (1962) Les troubles de la memoire accompagnant des lesions hippocampiques bilaterales. In Physiologie de l'Hippocampae, P. Passant, ed., pp. 257-272, Centre de la Recherche Scientifique, Paris.

Mishkin, M., and H. L. Petri (1984) Memories and habits: Some implications for the analysis of learning and retention. In Neuropsychology of Memory, N. Butters and L. R. Squire, eds., pp. 287-296, Guilford, New York.

Mitcham, J. C., and R. K. Thomas (1972) Effects of substantia nigra 
and caudate nucleus lesions on avoidance learning in rats. J. Comp. Physiol. Psychol. 81: 101-107.

Mitchell, J. A., S. Channel, and G. Hall (1985) Response-reinforcer associations after caudate-putamen lesions in the rat: Spatial discrimination and overshadowing-potentiation effects in instrumental learning. Behav. Neurosci. 99: 1074-1088.

Neill, D. B., and S. P. Grossman (1971) Behavioral effects of lesions or cholingergic blockade of the dorsal and ventral caudate of rats. $J$. Comp. Physiol. Psychol. 71: 311-317.

O'Keefe, J., and D. H. Dunway (1980) On the trail of the hippocampal engram. Physiol. Psychol. 8: 229-238.

O'Keefe, J., and L. Nadel (1978) The Hippocampus as a Cognitive Map. Oxford U. P., Oxford, UK.

O'Keefe, J., L. Nadel, S. Keightley, and D. Kill (1975) Fornix lesions selectively abolish place learning in the rat. Exp. Neurol. 48: 152166.

Olton, D. S. (1978) The function of septo-hippocampal connections in spatially organized behavior. Functions of the septo-hippocampal system. Ciba Foundation Symposium, Elsevier, Amsterdam.

Olton, D. S., and W. A. Feustle (1981) Hippocampal function required for nonspatial working memory. Exp. Brain Res. 41: 380-389.

Olton. D.S., and B.C. Papas (1979) Spatial memory and hippocampal function. Neuropsychologia 17: 669-682.

Olton, D. S., and R. J. Samuelson (1976) Remembrance of places passed: Spatial memory in rats. J. Exp. Psychol. [Anim. Behav. Proc.] 2: $97-115$

Olton, D. S., and P. Schlosberg (1978) Food-searching strategies in young rats: Win-shift predominates over win-stay. J. Comp. Physiol. Psychol. 92: 609-618.

Paxinos, G., and C. Watson (1982) The Rat Brain in Stereotaxic Coordinates, Academic, New York.

Peeke, H. V. S., and M. J. Herz (1971) Caudate nucleus stimulation retroactively impairs complex maze learning in the rat. Science 173 : $80-82$.

Pellegrino, L. J., and D. F. Clapp (1971) Limbic lesions and externally cued DRL performance. Physiol. Behav. 7: 863-868.

Port, R. L., A. L. Beggs, and M. M. Patterson (1987) Hippocampal substrate of sensory associations. Physiol. Behav. 39: 643-647.

Prado-Alcala, R. A., Z. J. Grinberg, Z. L. Arditti, M. M. Garcia, H. G. Prieto, and H. Brust-Carmona (1975) Learning deficits produced by chronic and reversible lesions of the corpus striatum in rats. Physiol. Behav. 15: 283-287.

Rickert, E. J., T. L. Bennet, G. J. Anderson, J. Corbett, and L. Smith (1973) Differential performance of hippocampally ablated rats on nondiscriminated and discriminated DRL schedules. Behav. Biol. 8: 597-609.

Ross, R. T., W. B. Orr, P. C. Holland, and T. W. Berger (1984) Hippocampectomy disrupts acquisition and retention of learned conditional responding. Behav. Neurosci. $98: 211-225$.

Samuels, I. (1972) Hippocampal lesions in the rat: Effects on spatial and visual habits. Physiol. Behav. 8: 1093-1098.

Schwartzbaum, J. S., and P. J. Donovick (1968) Discrimination reversal and spatial alternation associated with septal and caudate dysfunction in rats. J. Comp. Physiol. Psychol. 65:83-92.

Silveira, J. M., and D. P. Kimble (1968) Brightness discrimination and reversal in hippocampally-lesioned rats. Physiol. Behav. 3:625630.

Solomon, P. R., and J. W. Moore (1975) Latent inhibition and stimulus generalization of the classically conditioned nictitating membrane response in rabbits (oryctolagus cuniculus) following dorsal hippocampal ablation. J. Comp. Physiol. Psychol. 89: 1192-1203.

Stevens, R., and A. Cowey (1972) Enhanced alternation learning in hippocampectomized rats by means of added light cues. Brain Res. 46: $1-22$.

Sutherland, R. J., B. Kolb, and I. Q. Whishaw (1982) Spatial mapping: Definitive disruption by hippocampal or medial frontal cortex damage in the rat. Neurosci. Lett. 31: 271-276.

Suzuki, S., G. Augerinos, and A. H. Black (1980) Stimulus control of spatial behavior on the eight-arm maze in rats. Learn. Motiv. $11: 1-$ 18.

Thomas, G. J., and D. M. Gash (1985) Mammillothalamic tracts and representational memory. Behav. Neurosci. 99: 621-630.

Thorndike, E. I. (1933) A proof of the law of effect. Science 17: $173-$ 175.

Viaud, M., and N. M. White (1989) Disassociation of visual and olfactory conditioning in the neo-striatum of rats. Behav. Brain Res. 32: $31-42$.

Volpe, B. T., R. Johnson, J. Ellenberger, and H. P. Davis (1986) Performance of rats with either ischemic hippocampal injury, or bilateral radiofrequency lesions of the hippocampus or caudate on a 12-arm radial maze. Soc. Neurosci. Abstr. 12: 745.

Walker, D. W., L. W. Means, and R. I. Isaacson (1970) The effects of hippocampal and cortical lesions on single alternation go/no go acquisition in rats. Psychonomic. Sci. 21: 29-31.

Walker, J. A., and D. S. Olton (1984) Fimbria-fornix lesions impair spatial working memory but not cognitive mapping. Behav. Neurosci. 98: 226-242.

Whishaw, I. Q., and B. Kolb (1984) Decortication abolishes place but not cue learning in rats. Behav. Brain Res. 11: 123-134.

Whishaw, I. Q., G. Mittleman, S. T. Bunch, and S. B. Dunnet1 (1987) Impairments in the acquisition, retention, and selection of spatial navigation strategies after medial caudate-putamen lesions in rats. Behav. Brain Res. 24: 125-138.

Winocur, G. (1974) Functional dissociation within the caudate nucleus of rats. J. Comp. Physiol. Psychol. 86: 432-439.

Winocur, $G$. (1980) The hippocampus and cue utilization. Physiol Psychol. 8: 43-53.

Winocur, G., and C. B. Breckenridge (1973) Cue-dependent behavior of hippocampally damaged rats in a complex maze. J. Comp. Physiol. Psychol. 82: 512-522.

Wyers, E. J., and S. A. Deadwyler (1971) Duration and nature of retrograde amnesia produced by stimulation of the caudate nucleus. Physiol. Behav. 6: 97-103.

Wyers, E. J., H. V. S. Peeke, J. S. Williston, and M. J. Herz (1968) Retroactive impairment of passive avoidance learning by stimulation of the caudate nucleus. Exp. Neurol. 22: 350-366.

Zola-Morgan, S., L. R. Squire, and M. Mishkin (1982) The neuroanatomy of amnesia: Amygdala-hippocampus versus temporal stem. Science 218: 1337-1339. 